\title{
Zu diesem Buch
}

Wenn man auf die Frage nach dem Beruf Sprachwissenschaftlerin angibt, folgt fast immer eine typische Rückfrage, nämlich: für welche Sprachen denn? Für viele Menschen ist also durchaus plausibel, dass „fremde“ Sprachen beschrieben und erforscht werden müssen - Linguistik als eine Wissenschaft, die sich mit dem Funktionieren der eigenen Sprache beschäftigt, ist dagegen im öffentlichen Bewusstsein kaum präsent. Auf den ersten Blick ist das durchaus nachvollziehbar eigentlich leben wir ja mit „unserer“ Sprache sozusagen einvernehmlich. Unser sprachlicher Alltag verlangt nur selten danach, über die Weisen nachzudenken, wie Sprache ihren Dienst tut, oder gar, welche Elemente und Prozesse dabei im Einzelnen eine Rolle spielen. Schon eher fällt uns ihre Wirksamkeit auf: wie ein einziger Satz - oder sogar nur ein Wort - uns einen Zusammenhang erkennen lässt, uns nachdenklich macht oder uns ärgern, freuen und sogar verletzen kann. Das zeigt, dass Sprache einen sehr direkten Zugang zu unserem Denken und Fühlen hat. Und es führt uns im nächsten Schritt zu der Frage, wie diese Verbindung möglich wird. Ein allgemeines Ziel von Sprachbeschreibung kann also sein, die Vermittlungswege zwischen Denken, Fühlen, Handeln und Sprechen zu verfolgen.

Ein wesentlicher Aspekt dieses Ziels ist es, Verständigungsprozesse nachvollziehbar zu machen. Das kann einerseits Gespräche betreffen. Wir machen immer wieder die Erfahrung, dass Fehl- und Missverständnisse gerade dann häufiger auftreten und weitreichende Folgen haben, wenn gegenseitiges Verstehen besonders wichtig wäre, z.B. im Streit oder in Gesprächen, in denen viel auf dem Spiel steht. Kann man dem vorbeugen - und auf welche Weise? Um darauf eine konstruktive Antwort zu finden, die sich nicht nur auf den einzelnen Fall beschränkt, müssen wir verstehen lernen, wie Gespräche funktionieren: Welche Strukturen wiederholen sich in ihrem Verlauf, welche typischen Funktionen werden mit welchen sprachlichen Mitteln erfüllt und welche Rolle kommt z.B. der Beziehung zwischen den Gesprächspartnern dabei zu? (dazu Kap. 4 und 11). Es geht also darum, grundsätzliche Prinzipien zu klären, die für den Ablauf von Gesprächen gelten.

Sprachliche Verständigungsprozesse prägen aber auch das Zusammenleben in der Gesellschaft, also auf einer über-individuellen Ebene. Wie z.B. ein politischer Sachverhalt in den Medien sprachlich dargestellt wird, ist oft entscheidend dafür, wie wir ihn „,sehen“ - und entsprechend, wie wir darüber denken und vielleicht in der Folge handeln. Es macht einen Unterschied, ob wir von Ausländern oder Flüchtlingen, von Asylbewerbern oder Migrantinnen sprechen, denn in jeder dieser Bezeichnungen steckt eine spezifische Perspektive, wird jeweils ein Aspekt des komplexen Zusammenhangs fokussiert, andere bleiben im Hintergrund 
(Kap. 3). Zur Ausleuchtung solcher Prozesse tragen verschiedene Bereiche der Linguistik bei: Semantik analysiert, wie Bedeutung grundsätzlich „zustande kommt“ (Kap. 10) und wie sie in der Interaktion verändert werden kann (Kap. 11). In der Soziolinguistik und der Diskursanalyse wird u.a. untersucht, wie die Herkunft und die Rollen von Beteiligten und gegebenenfalls die entsprechenden Machtverhältnisse zwischen ihnen eine Argumentation beeinflussen können (Kap. 12). Zusammengefasst: Ein tieferes Verständnis sowohl individueller als auch gesellschaftlicher Verständigungsprozesse setzt eine detaillierte Beschreibung der dabei verwendeten sprachlichen Formen und Strategien voraus. Langfristiges Ziel ist dabei, die Wirkung der jeweils verwendeten sprachlichen Mittel zu erklären.

Ein zweiter Bereich, in dem wir auf das Wirken der Sprache gestoßen werden, sind Texte: solche, die wir lesen, und solche, die wir schreiben, z. B. in der Schule oder im Studium. Texte als Lese-Aufgabe können auf verschiedene Weise herausfordernd sein, denn auch die Ziele bei ihrer Rezeption sind jeweils verschieden: Die Bedienungsanleitung soll vor allem ermöglichen, mit einem neuen Gerät zurechtzukommen; der wissenschaftliche Aufsatz dagegen zielt z.B. darauf, neue begriffliche Unterscheidungen in einem spezifischen Themenbereich nachvollziehbar zu machen. Die typischen Schwierigkeiten beim Verständnis in diesen beiden Textsorten (und vielen weiteren Fällen) werden durchschaubarer, wenn wir einerseits unser Wissen über den Bau und die innere Organisation von Texten erweitern (Kap. 8, 9) und andererseits verstehen, welche Prozessschritte beim Lesen nacheinander bearbeitet werden (Kap. 5).

Ein Verständnis vom Text als einem Gewebe, das auf vielschichtige Weise strukturiert ist und in hohem Maße auf den Einbezug von mitgebrachtem Wissen baut (Kap. 3), kann aber auch im Fall der Textproduktion, also beim Schreiben, nützlich werden - wenn wir z.B. vor dem viel besprochenen „leeren Blatt“ sitzen und einen ersten Satz vielleicht schon einige Male wieder gelöscht haben. Es kann Bezugspunkt dafür sein, Texte in begründeter Weise zu beurteilen, zu vergleichen, zu verbessern. Die These ist also: Linguistisches Wissen hilft beim „fortgeschrittenen“ Lesen und Schreiben - indem es eine Modellierung für die Einzelschritte und Komponenten anbietet, die dabei eine Rolle spielen.

Drittens kann aus der genauen Beschreibung sprachlicher Formen und ihrer Verwendungskontexte noch ein weiterer Erkenntnisgewinn erwachsen: Linguistische Beschreibung macht erfahrbar, dass Sprache immer in Veränderung begriffen ist - und dass das zu ihrer Natur gehört (Kap. 2). Jedes einzelne Wort, jede Routineformel, jede Textsorte, jeder Gesprächstyp existiert typischerweise in vielen Varianten, hat eine Entstehungsgeschichte und wird sich weiterentwickeln, verändern, und vielleicht auch wieder aus dem Gebrauch verschwinden. Nichts im Bereich des Sprachlichen war „schon immer so“ oder wird unverändert immer so bleiben. 
Die Einflüsse, die solche Veränderungen anstoßen und weiterbringen, kommen aus vielen Quellen: aus dem Sprachverhalten des Einzelnen, aus der sozialen Interaktion, und auch aus der Dynamik der Sprache selbst. Wollen wir z.B. die Entwicklung eines Dialekts nachzeichnen, müssen wir zunächst beschreiben, wie er sich durch ein bestimmtes Bündel von sprachlichen Merkmalen von anderen Sprachformen unterscheidet: Die Aussprache ist dabei ein wichtiger Aspekt, vielleicht aber auch der Wortschatz (Kap. 6) oder bestimmte Eigenheiten der Grammatik (Kap. 7, 8). Um Veränderungen erklären zu können, müssen aber auch Verwendungskontexte berücksichtigt werden: Welche Sprecher verwenden diesen Dialekt bei welchen Gelegenheiten - und wie verändert sich das? Erst durch einen genauen Blick darauf, welche Sprachform in welchen Situationen von welchen Sprechern tatsächlich gebraucht wird, können wir z.B. einschätzen, ob ein Dialekt grundsätzlich auf dem Rückzug ist, oder doch in bestimmten Bereichen ,weiterlebt“.

Die wissenschaftliche Beschreibung von Sprache hat damit auch eine Art Spiegelfunktion - denn wir selbst sind von der Beteiligung an solchen Entwicklungen ja nicht ausgenommen. Durch den linguistischen Blick kann uns bewusst werden: genaugenommen trägt jede einzelne Sprachverwendung zum „Haushalt“ unserer Sprache bei - sei es stabilisierend oder verändernd. So können wir uns im Alltag oft entscheiden, ob wir ein neues englisches oder ein herkömmliches deutsches Wort für einen Sachverhalt verwenden wollen. Wir können uns entscheiden, nie mehr das generische Maskulinum zu verwenden, oder in Zukunft beim privaten Schreiben auf Großbuchstaben ganz zu verzichten. Kurz: Zu erkennen, dass Sprache eine „so gewordene“ Sprache ist, bedeutet auch, sie als veränderbar zu erkennen. Auf eine Formel gebracht: Die Sprache sind wir!

Am Ende jedes Kapitels geben wir einige wenige Literaturhinweise zum Weiterlesen. Alle anderen Texte, aus denen wir zitieren oder auf die wir im Laufe dieses Buchens verweisen, nennen wir am Ende des Buchs im Literaturverzeichnis.

Wir haben uns bemüht, bei unserer Darstellung zu berücksichtigen, dass Sprache immer im menschlichen Handeln, der Interaktion und in gesellschaftlichen Situationen verankert ist und auch so verstanden werden muss. In unserem Buch gibt es deshalb kein eigenes Kapitel zur Pragmatik. Das ist die linguistische Teildisziplin, die erklärt, wie man mit Sprache handelt und welche Beziehung Sprache zum Kontext ihrer Verwendung hat. Das unterscheidet es von den meisten anderen Einführungen in die Linguistik. Wir denken, dass Pragmatik ein fundamentaler Gesichtspunkt aller sprachlicher Phänomene ist, und haben unser Buch entsprechend so aufgebaut, dass pragmatische Modelle und Erklärungen den roten Faden bilden.

Wir danken den Studierenden sowie den Kolleginnen und Kollegen in Heidelberg und Darmstadt, die kapitelweise oder auch im Ganzen Vorversionen dieser 
Einführung gelesen und uns wertvolle Anregungen gegeben haben. Carina Kiemes und Mira Winkelbrandt danken wir für Recherchen, das Lektorat und die Einrichtung des Manuskripts. Unser Dank gilt auch Ekkehard Felder und der weiteren anonymen Person, die unser Buch für den Verlag begutachtet haben, für viele Anregungen und Hinweise. Verbliebene Fehler und Ungereimtheiten sind allein uns anzulasten. Dem Verlag De Gruyter, namentlich Carolin Eckardt, Albina Töws und Andreas Brandmair, danken wir für die Betreuung und die Aufnahme in die Reihe De Gruyter Studienbuch.

Was die Darstellung der sozialen Geschlechter (Gender) in der Sprache betrifft, so haben wir uns dafür entschieden, dort Doppelnennungen (Leserinnen und Leser) zu verwenden, wo es der Schreibfluss zulässt, und ansonsten, z. B. bei Aufzählungen, zu alternieren (z.B. Verlegerinnen, Autoren, Lektorinnen und Kriti$k e r)$. Wir sind uns darüber bewusst, dass diese Lösung nicht alle Leserinnen und Leser überzeugen wird, für uns ist sie aber in diesem Moment der Sprachgeschichte die stimmigste. In jedem Fall meinen wir alle, die wissbegierig sind und Lust haben, sich mit der Sprache zu beschäftigen - der alläglichsten Sache der Welt, die uns in dem Maße beherrscht, in dem wir sie beherrschen; die unsere Erfahrungen, Ideen und Erinnerungen prägt und ohne die wir nicht die wären, die wir sind. 